\title{
LA RECEPCIÓN DE LAS NOVELAS DE ZOLA Y DE PARDO BAZÁN EN LA PRENSA ESPAÑOLA DE LA ÉPOCA
}

\author{
František Dratva
}

\begin{abstract}
The article deals with the reception of Émile Zola's novels and Emilia Pardo Bazán's Los pazos de Ulloa in Spanish press in the late nineteenth century. In each case, five articles were taken as a source and the intention was to reflect the most important opinions and their developments.

Keywords: Émile Zola; Emilia Pardo Bazán; Naturalism; Reception; Press.

Resumen: El artículo analiza la recepción que tuvieron en los periódicos españoles de finales del siglo XIX las novelas de Émile Zola y Los pazos de Ulloa de Emilia Pardo Bazán. En cada caso se han tomado como fuente cinco artículos y se ha pretendido reflejar las opiniones más relevantes y la evolución de las mismas.
\end{abstract}

Palabras clave: Émile Zola; Emilia Pardo Bazán; Naturalismo; Recepción; Prensa.

\section{Nota introductoria}

En el presente trabajo se analizará cómo fueron recibidas en España las novelas de Émile Zola durante el periodo comprendido entre 1882 y 1890, y cómo fue acogida la novela Los pazos de Ulloa en los años 1886 y 1887. En el primer caso se tomarán como base los artículos publicados en el periódico El Imparcial - para mostrar la evolución y divergencia de las opiniones en la misma publicación - ; en el segundo se recurrirá a los artículos aparecidos en El Liberal, La Dinastía, La Ilustración Ibérica y Galicia. Todas las reseñas han sido consultadas en la página web de la Hemeroteca Virtual, que forma parte de la Biblioteca Nacional de España.

\section{La recepción de las novelas de Émile Zola}

El primer artículo seleccionado que refleja la recepción generada por las novelas de Zola se titula "París", es del 30 de enero del año 1882 y no figura en él el nombre del autor. Se trata de un análisis del libro Pot-Bouille, que fue traducido al español al año siguiente bajo el título Miseria humana (Pattinson 1969: 53). Al principio se menciona que la popularidad del escritor galo fue tan notoria que varios periódicos franceses compitieron para poder editar la obra como folletín. El autor alude también a la circunstancia de que el periodista y político Rochefort pidió a Zola que le permitiera publicar Pot-Bouille en su 
diario L'Intransigeant de una manera gratuita, ya que según el político francés "vuestra novela se propone el mismo fin político y social que mi periódico". Zola rechazó esta propuesta; el articulista lo describe de la siguiente manera: "un hombre sereno, frío, endurecido por los engaños", refiriéndose al poco éxito de las empresas literarias de Zola previas a Thérèse Raquin y haciendo una valoración personal del carácter del padre del Naturalismo (Anónimo 1882).

Tras una sucinta información sobre el argumento, el articulista proclama que para el naturalista el tema es lo de menos. Comenta que Zola concede poca importancia al "interés vulgar" de preparar sorpresas para el lector cambiando el orden de las escenas. Es interesante también la referencia al hecho de que tan solo una tercera parte de los lectores españoles que decían leer a Zola lo hacían realmente. Esto apoya la tesis de Clarín, quien se quejaba de que muchos de los que expresaban sus opiniones acerca del Naturalismo no habían leído ninguna obra de Zola, y se basaban en lo que habían oído decir sobre ellas (Pardo Bazán 1989: 11).

Se cita al escritor Amicis, que describió el estilo del autor de Naná de la siguiente manera:

¿Sois lectores de Zola? Pues os obligará a seguirle a donde se le antoje ir. No respetará vuestra edad ni vuestra educación; os arrastrará al figón, a la taberna, a la casa de juego; os hará asistir al baile en el salón, a la escena de celos en un lupanar, a la embriaguez de una taberna (Anónimo 1882).

Otra referencia biográfica que se menciona en el artículo subraya el hecho de que Émile Zola estaba casado por el rito católico; además le unía con su mujer el vínculo de los días de pobreza, y añade que era un marido ejemplar. Se diría que el crítico pretende paliar las iras que despertaron la temática y la ambientación de la novelas zolescas. La valoración de estas que aparece en "París" es completamente laudatoria. El articulista acaba afirmando que Zola "tiene los dos caracteres que dan la victoria en las luchas del arte: talento y paciencia" (Anónimo 1882).

La evaluación que hace de Zola Jacinto Octavio Picón en la recensión "La última novela de Zola" ya no es tan favorable; no obstante, todavía encuentra en la obra del novelista francés más elementos positivos que negativos. Picón teoriza sobre el origen del Naturalismo y defiende que el verdadero fundador de la nueva escuela literaria fue Gustave Flaubert, relegando a Zola al papel del propagandista de la misma.

Picón considera importante resumir los principales rasgos del movimiento, como por ejemplo el análisis de la moral de los personajes, examen psicológico, lucha entre la lógica y la pasión, choque del libre albedrío con las imposiciones de la realidad y la determinación (Picón 1883). Más tarde será Pardo Bazán quien, en La cuestión palpitante, lleve a cabo esta sistematización de una manera más profunda.

"Ha exagerado algo la tendencia [naturalista]" afirma Picón acerca de Zola, aunque lo justifica haciendo hincapié en el papel que, según él, ocupa el novelista en dicha corriente. Resalta que, mientras que en el pasado un autor necesitaba solamente la imaginación, el escritor de finales del siglo XIX precisaba también de dotes de observación, de pensamiento y de poesía que embellecieran la realidad. Como prototipo de este nuevo novelista vuelve a mencionar a Flaubert, criticando a Zola por llevar al extremo algunos de estos procedimientos y eliminar otros. Se refiere a que, en las obras del segundo autor, la observación gana terreno a costa de la imaginación. La poesía - que acicala la realidad - apenas aparece, al igual que ocurre en la vida real. Lo físico y lo moral tienen en 
las obras de Zola la misma importancia que en la vida, y así lo aprecia Picón, estimando el realismo y la capacidad de observación. Sin embargo, le critica por sus descripciones, a veces demasiado meticulosas.

Posteriormente, Jacinto Picón se opone a los que atacan el pesimismo de Zola y hasta le llega a otorgar a este intenciones moralistas, pues "quien pone al descubierto lo censurable, da el primer paso para corregirlo." Ejemplifica su opinión enumerando los vicios que aparecen en las anteriores composiciones de Zola (Picón 1883). A continuación valora la capacidad del novelista francés de reflejar la sociedad de su tiempo y le equipara con los máximos representantes de la novela española. Expresa su sorpresa por el rechazo que existe hacia Zola en el país que dio al mundo tan preciosas muestras de realismo como los personajes de Sancho Panza o Lázaro de Tormes. Constituye la mayor parte del artículo el resumen de la recién publicada novela $A u$ bonheur des dames, en donde se menciona la presencia de escenas de la "miseria más repulsiva" o la "prostitución más descarada", pero también de "escenas maravillosas", no tanto por el argumento como por la manera de narrar la acción.

Picón considera que los mayores encantos de la obra son los elaborados personajes y el realismo. Su único defecto se encuentra en que las descripciones - a pesar de estar admirablemente compuestas - pecan de pesadas, por lo que Au bonheur des dames se puede convertir, para muchos, en un libro enojoso. No obstante, reitera los halagos referentes a las dotes observadoras de Zola.

El tercer artículo, al igual que el primero, carece de firma y se refiere a la publicación de Germinal. A diferencia de los anteriores, en este caso no se dedicó a la nueva novela de Zola un artículo entero, sino solo una parte del texto llamado "Bibliografía", lo que nos hace pensar que la nueva obra del literato francés ya no fue recibida con igual entusiasmo que las anteriores. Efectivamente: el periodista, después de afirmar que en París se prohibió la representación del drama basado en Germinal, advierte que allí "abundan las escenas más asquerosamente naturalistas" (Anónimo 1885). Esta calificación muestra que ya en el año 1885, antes de que apareciese Los pazos de Ulloa de Pardo Bazán, el Naturalismo francés fue recibido, a veces, con rechazo.

El autor de "Bibliografía" encarece, al igual que los autores de las reseñas anteriormente citadas, el espíritu de observación del novelista francés. Para sentenciar la mención que hace Zola de los aspectos más míseros de la vida, recurriendo a un léxico próximo a la jerga, el crítico utiliza la siguiente metáfora: "Lo lamentable es que, para describir todo eso y describirlo admirablemente, Zola haya creído necesario con desconsoladora frecuencia mojar su pluma en el fango" (Anónimo 1885). La apreciación final sobre Germinal es una cruel crítica de la entonces recién publicada novela: "Con ellas [las hediondeces] quedará relegado al fondo en que las personas de buen gusto y de sentido recto arrojan los libros pornográficos". La ofensa del buen gusto en las composiciones naturalistas fue uno de los principales reproches de los críticos y periodistas que abominaban de este movimiento literario.

El artículo de Carlos Malagarriga publicado en El Imparcial el 9 de septiembre de 1888 se vuelve a titular "La última obra de Zola", y su extensión es relativamente grande: ocupa la mitad de la página. Parece plausible que esto se deba a que la novela analizada, Le Rêve, difiere bastante de Germinal. Se trata de una composición que refleja el misticismo presente entre los creyentes franceses ya muy avanzado el siglo XIX, y no aparecen escenas llenas de "hediondeces", como diría el autor del artículo del año 1885. Malaga- 
rriga está de acuerdo con los editores del libro, que afirman que Le Rêve es una narración completamente casta; discrepa no obstante con los que la consideran una obra insípida.

Para dar muestras de que no se trata de un hecho excepcional, de que Zola ya varias veces escribió una novela "pulcra" después de otra que cumplía totalmente con los presupuestos naturalistas, alega el caso de la publicación de la idílica obra La Faute de l'abbé Mouret, tras las creaciones que "incitaron contra su autor las iras de los partidarios de la literatura del justo medio". Podemos comprender el justo medio, en este caso, como sinónimo de bon goût, cuyo antónimo está mencionado en el párrafo en que Malagarriga declara que Une page d'amour fue escrita para "tranquilizar a los que creían a Zola definitivamente perdido en la corriente del mal gusto". En cuanto a las novelas analizadas en este trabajo, Malagarriga afirma que Pot-Bouille parecía la declaración de guerra a la clase media, mientras que $A u$ bonheur des dames cantaba las grandezas de la industria moderna (Malagarriga 1888). Tras la afirmación de que Zola es un creador que volvía a probar la elasticidad de su talento y la imparcialidad de su obra, lo compara - tal como lo había hecho Picón - con los grandes genios de la literatura, por ejemplo con Cervantes, Calderón, Shakespeare o Balzac.

Para concluir, reitera que tal vez se sientan decepcionados los que estén esperando de Le Rêve el elemento brutal, arriesgado o atrevido que encontraron en las anteriores creaciones del padre del Naturalismo, pero que seguramente la recibirán con mucho gusto los que aprecian su labor honda y humana y estiman la pasión que siente por los débiles. En el mismo final del artículo califica a Zola como el primer novelista de su tiempo. Teniendo en cuenta que las únicas críticas que aparecen en este artículo son las que hacen referencia a que algunas de las obras de Zola son demasiado naturalistas, podemos afirmar que la valoración que se hace del autor francés es muy positiva.

El último artículo que refleja la recepción de las novelas de Émile Zola en periódicos españoles entre los años 1882 y 1890 es del mismo director de Los Lunes de Imparcial: José Ortega Munilla. Fue publicado el 7 de abril de 1890, se titula "La última novela de Zola" y su extensión es la más amplia de todos los textos analizados en este trabajo; sin embargo, la crítica que se hace de Zola no resulta nada favorable.

Tras la alabanza del valor social de que goza la literatura en Francia, donde la publicación de una novela de un autor de renombre despierta los más vivos intereses, Ortega Munilla comenta el hecho de que los novelistas franceses - Zola incluido - adquieren una fortuna considerable gracias a su producción literaria (Ortega Munilla 1890). La mención de este dato ya apareció en el artículo del año 1882. En ello podemos observar la importancia que le concedían los periodistas españoles al factor socioeconómico de las bellas letras francesas, siendo este más notable que en el ámbito español.

Al igual que Picón, Ortega Munilla analiza los constituyentes de la obra de Zola, aunque difiere de él porque se fija más en el lado científico del Naturalismo que en otros aspectos. Alude a que el creador de Germinal aplica la fisiología de Claudio Bernard y busca la causa física de cada sensación. Según su criterio, esto es posible solo si lo lleva a cabo alguien como Zola: un hombre que posee ilustración variadísima, penetración perspicaz y clarividente. No obstante, en este momento terminan las alabanzas. En referencia a la última obra del autor francés, el periodista advierte de que Zola ha exagerado su sistema y "el médico, el alienista, el fisiólogo, ocupan un lugar más importante que el poeta y el novelista". 
Otro reproche es que Zola ha dejado de describir los asuntos cotidianos y que se ha empezado a interesar por lo insólito: "se ha perdido en el laberinto de lo excepcional" y "se ha convertido en el narrador de casos teratológicos" (Ortega Munilla 1890). Todo esto surge a raíz del análisis del protagonista de la obra reseñada en el artículo: La Bête humaine. Es un conductor de trenes, llamado Jacques Lantier, que de cuando en cuando siente un irrefrenable deseo de matar. Ortega arremete duramente contra este tipo de argumento preguntando: " ¿Es que Zola se ha propuesto representar en La bestia humana el lado animal, fisiológico e instintivo que hay dentro del ser racional?” y “ ¿Es que todos nosotros llevamos dentro de nuestra levita de hombres civilizados este demonio de la carne irascible y sangrienta?" La respuesta del periodista no puede ser más clara: "No, y cien veces no."

Posteriormente, vuelve a criticar el argumento de la narración por no existir referentes de tales hechos en la vida cotidiana, salvo la excepción de algún que otro caso sucedido en un manicomio. Por salirse el tema de La Bête humaine de lo habitual, pierde, según el crítico, todo interés literario, y el único grupo al que puede interesar es al de los médicos, en ningún caso los lectores de novelas. Ortega Munilla intenta dejar su opinión aún más clara afirmando que "es preciso que el literato se atenga a la realidad" y que "cuanto más verdadera es la escena descrita, más literaria ha de resultar la página en que se describa". Cuán sorprendido habría quedado el padre de Ortega y Gasset si hubiera sabido que, tan solo un cuarto de siglo más tarde, el que es considerado por muchos el mayor novelista del siglo XX escribiría la historia de un hombre que se convirtió en mosca.

Podemos constatar el siguiente reproche a la novela en la dura oposición que expresa a que un novelista pinte de una manera pormenorizada el desarrollo del cáncer en el rostro de un hombre, pues considera estas descripciones de mal gusto y las relega otra vez al campo de la medicina. Al igual que Amicis, citado en el artículo del año 1882, Ortega Munilla sentencia que Zola obliga al lector a asistir a escenas que califica de inadecuadas para el público. Con una crítica parecida se encontró Emilia Pardo Bazán en el año 1884, ya que Luis Alfonso le reprochó que en su obra La tribuna ofreciera una minuciosa descripción de un parto (Pardo Bazán 1989: 374).

Más adelante, el crítico vuelve a insistir en que la historia narrada es un caso excepcional, y aduce el ejemplo reciente de un hombre que se había presentado en la gendarmería pidiendo que le detuvieran, ya que, según dijo él mismo, tenía inmensas ganas de derramar sangre humana y particularmente la de sus propios hijos. El individuo fue llevado al manicomio, solución que recomienda don José para el protagonista de La bestia humana: "sacarle del libro, sacarle de la literatura y encerrarle en una casa de locos".

Para subrayar cómo Zola se atenía a la realidad en sus obras anteriores, menciona entre otras la novela analizada en el artículo de 1882, en que es patente "el egoísmo hipócrita de los burgueses, sirviendo de capa a los crímenes" (Ortega Munilla 1890), o Germinal, en que se vio a los mineros sometidos a la esclavitud experimentar "impulsos de indignación contra el capitalista". Y puesto que Ortega Munilla considera Germinal una buena novela, no critica las descripciones de hediondeces que en ella aparecen, como hizo el autor de "Bibliografía" de 1885 (Anónimo 1885).

El único momento en que el periodista alaba la última creación de Zola es cuando menciona el inmenso talento del francés para la observación, por ejemplo a la hora de describir un temporal de nieve con que lucha el joven conductor de tren. Al leer aquel pasaje, el lector experimenta "el frío, la incomodidad y hasta el terror de la catástrofe." Al 
igual que los articulistas anteriores, compara a Zola con los grandes maestros de la literatura universal, con Shakespeare y Hugo en este caso, pero no para equipararlo a ellos, sino para reivindicar que sabían crear personajes extraordinarios - Hamlet y Cuasimodo - sin que la moral se viese perjudicada.

\section{La recepción de Los pazos de Ulloa}

La primera reseña, según el orden cronológico, que muestra la recepción de Los pazos de Ulloa de Emilia Pardo Bazán es el homónimo artículo de Miguel Moya, publicado el 28 de octubre del año 1886 en El Liberal. En aquel momento todavía no había sido editado el segundo de los dos tomos de los que consta la narración, por lo que el autor se dedica principalmente al análisis de los Apuntes autobiográficos de doña Emilia que precedían el primer tomo. El contenido de estas notas biográficas y la valoración de las mismas por parte de Moya nos proporcionan informaciones interesantes sobre la polémica del Naturalismo; no obstante, este no es el tema del presente trabajo. Para nuestro fin se aprovechan dos breves juicios sobre el primer tomo de la obra, primero cuando Moya habla de "las bellezas de este primer tomo" y segundo cuando señala el hecho de que Pardo Bazán "da a nuestra admiración novelas como Un Viaje de Novios, La Tribuna y Los pazos de Ulloa" (Moya 1886).

En enero de 1887 apareció en La Coruña el primer número de la revista Galicia, que contiene un análisis del primer tomo de Los pazos de Ulloa escrito por Ramón Segade Campoamor. Ya el comienzo del mencionado artículo nos hace sospechar que la valoración no va a ser del todo favorable: "Sería pretensión indisculpable echarnos aquí de críticos" y "a cualquier hijo de vecino que anduvo y anda metido entre libros toda la vida, puede permitírsele diga lo que le parece y sacó en limpio del último que cayó en sus manos."

Analizando los Apuntes autobiográficos, Segade estima que doña Emilia es mujer de dotes extraordinarias y que enriqueció su entendimiento con valiosoa erudición (Segade Campoamor 1887: 40). Posteriormente, se prepara el campo para las futuras críticas, afirmando irónicamente que:

...la Providencia da y concede y comunica a las criaturas [...] tan soberanas dotes de inteligencia y dosis de buen sentido, que aun en el mayor peligro en que la inteligencia más firme puede hallarse, la preserva y libra de perderse en concepciones extrañas...

Y más adelante: "una madre celosa y verdaderamente cristiana, no podía ser llevada por insanas teorías".

Continuando con el comentario de la biografía de Pardo Bazán, el periodista menciona que, después de la Revolución de Septiembre, doña Emilia se fue al extranjero en busca de un ambiente que estuviera en armonía con sus ideales y aficiones artísticas. El primero de los destinos mencionados es París, lo que nos hace pensar que Segade Campoamor puede estar criticando a la novelista gallega por importación de ideas extranjeras. Posteriormente, el periodista comenta los pasajes de los Apuntes bibliográficos que se refieren a La cuestión palpitante, en que doña Emilia expresa sus intenciones de descubrir y rechazar los elementos heréticos, deterministas y fatalistas del Naturalismo, intentando llevar a cabo un sincretismo que deje a salvo la fe. El autor gallego opina que la estética naturalista es incompatible tanto con la fe como con la moral, por lo cual "no hay ni habrá acuerdo posible". El culpable de todo esto es Zola, quien se sirvió del arte para propagar el más grosero materialismo (Segade Campoamor 1887: 44). 
El que, según Segade Campoamor, se dio cuenta de los principales errores de la corriente es Menéndez Pelayo, con quien desea que se una la novelista coruñesa. Así lo explica en el último párrafo de esta parte del artículo:

Perdónenos la ilustre escritora, la amiga estimadísima, esta manera franca de exponer nuestras opiniones; sabe cuánto la admiramos y respetamos por su talento y por su saber; pero no podemos sobreponernos a nuestros ideales ni hacerles traición por nada en este mundo.

Incluso llega a hablar de los seguidores de Zola como de "perniciosa secta de naturalistas franceses". Haciendo referencia a la parte final de la biografía de Pardo Bazán, en que esta describe su vida en la Granja en compañía de sus hijos, vuelve a abogar porque la escritora no siga los principios del Naturalismo, ya que si sabe describir tan bien lo real, lo hermoso, no hay necesidad de bajar a describir lo feo, triste y negro de la vida.

La valoración de la novela se ve marcada por las ideas conservadoras del periodista. Aprecia "el bellísimo libro" por su realismo, la buena caracterización de los personajes o la descripción de las costumbres gallegas, pero protesta contra la manera en que son presentadas las primitas del marqués de Ulloa: se trata de una visión cruda y positivista. En su opinión, la tierra gallega ofrece al literato muchas oportunidades para componer una novela ideal, hasta fantástica, sin entrar en discordia con la realidad (Segade Campoamor 1887: 48).

La última crítica tiene que ver con que doña Emilia siga el postulado naturalista de acuerdo con el cual el creador no se debe dejar notar en la obra. Para Segade Campoamor hacen falta estimaciones moralistas que ayuden al lector a comprender la enseñanza moral, de la cual un novelista nunca debería desentenderse.

Un punto de vista bien distinto es el que ofrecen los dos artículos - en realidad un artículo divido en dos partes - escritos por Clarín y publicados en La Ilustración Ibérica en el mes de febrero de 1887. Hay que tener en cuenta que fueron redactados antes de que se produjeran las divergencias entre su autor y Pardo Bazán (Pardo Bazán 1989: 355); de otra manera no hubiera sido posible que Clarín la llamase "mi buena amiga". Aprecia la aparición de Los pazos de Ulloa como una prueba de que la escritora gallega es diestra a la hora de elaborar buenas novelas.

Advierte que doña Emilia siempre ha descrito el campo gallego con maestría, y sin embargo nunca lo había hecho con la perfección que alcanza en la novela analizada. Subraya también que para ello es preciso "que el escritor conozca la vida rústica y sobre todo haya sentido los efluvios de su encanto infalible" (Clarín 1887a). Opina que doña Emilia sabe amar el terruño y ver la poesía útil del campo. A diferencia de Segade Campoamor, admira que la escritora sea capaz de describir "a la pobre Galicia con toda su miseria y con toda su hermosura natural", y tanto en la inmoralidad como en las grandezas espirituales de los que habitan esta tierra. Otro punto en que Clarín difiere del crítico gallego es que proclama que Pardo Bazán tal vez no abandone ni un momento la visión de lo bello, lo que la distingue de los que solo copian la realidad, de los escribientes.

Toma en buena consideración la forma en que retrata los personajes - tanto los principales como los secundarios - y el ambiente, al igual que la sucesión verosímil de los acontecimientos, junto con una sabia gradación de la acción. Aprecia el realismo, patente no solo en la construcción de los caracteres, sino también, por ejemplo, en la descripción de las cacerías y de sus preparativos correspondientes.

Según Clarín, el personaje mejor trabajado es Julián, ya que doña Emilia ha logrado convertirle en un héroe muy poético e interesante sin atribuirle ningún género de robus- 
tez (Clarín 1887b), consiguiendo que el lector empiece a prestar atención a lo que le está pasando al joven sacerdote. Compara al personaje principal de la novela con Hamlet, al igual que lo hiciera Ortega Munilla en el caso de La Bête humaine. En esta ocasión, sin embargo, se trata de una comparación positiva, tal como lo es todo el análisis de Los pazos de Ulloa realizado por Clarín.

El último artículo de nuestro corpus fue publicado en otoño del año 1887 en La Dinastía y su autor es Luis Alfonso. En este lugar hay que señalar que Luis Alfonso era fuerte opositor de Pardo Bazán, con quien en 1884 había mantenido una especie de querella epistolar (Pardo Bazán 1989: 350-391). En el mismo principio del análisis advierte: "Resaltan en esta obra los mismos defectos - defectos de escuela - que en las anteriores de la señora Pardo Bazán." Se refiere, en concreto, a la predilección por tipos y escenas vulgares, escasez de asunto, abundancia de episodios y pormenores y predominio de la materia sobre el espíritu (Alfonso 1887). Errores lingüísticos que ha encontrado en la narración son el uso del pretérito perfecto de subjuntivo hablando de asuntos pasados y el uso del verbo "deber" sin la preposición "de" en casos en que no se trata de una obligación precisa.

A continuación, procede a comparar la nueva novela con las anteriores de doña Emilia, y el juicio no es muy positivo. Afirma que Pardo Bazán es la mejor escritora española de todos los tiempos; no obstante, teniendo en cuenta que doña Emilia no quería que se la calificara en función de su condición femenina, sino según el valor de su creación literaria, creo que esta apreciación no le resultaría muy laudatoria.

El único momento en que podemos apreciar cierto elogio es cuando Luis Alfonso proclama que tanto los personajes como los sucesos están narrados de una manera viva y realista.

\section{Conclusión}

Tal como se ha pretendido demostrar en este trabajo, en las reseñas de las novelas de Émile Zola publicadas en los años analizados podemos notar que se produjo un cambio en la recepción de sus obras, ya que, con el paso de tiempo, esta fue cada vez menos entusiasta, con la excepción de la que se ocupa de Le Rêve. Esto, en mi opinión, se debe a la índole de la novela, ya que las más convencionales fueron mucho mejor considerados que las que cumplían con los postulados del Naturalismo. De igual manera, es necesario considerar que, muchas veces, el criterio personal del autor - mayor o menor grado de conservadurismo ideológico - de uno u otro artículo influyó sustancialmente en la valoración de la novela.

Por otra parte, las críticas a Los pazos de Ulloa fueron un claro reflejo de la recepción del Naturalismo y de La cuestión palpitante. Los que rechazaban y criticaban el Naturalismo francés actuaron de la misma manera a la hora de valorar el Naturalismo moderado de Emilia Pardo Bazán, mientras que los que estaban de acuerdo con los principios de este movimiento - o lo llevaban a la práctica literaria, como en el caso de Clarín - aplaudían las creaciones de la escritora gallega. También hay que tener en cuenta, como se ha mencionado anteriormente, que las relaciones personales entre Pardo Bazán y los críticos literarios, y la evolución de dichas relaciones, determinaron de una manera decisiva la crítica inmediata surgida en tiempos de la novelista. 


\section{Bibliografía:}

Alas Clarín, Leopoldo (1887a), «Los pazos de Ulloa», La Ilustración Ibérica 213, 6-7 [en línea], <http://hemerotecadigital.bne.es/datos1/numeros/ internet / Barcelona/Ilustración \% 20ibérica, \% 20La \% 20(Barcelona. \% 20 1883)/1887/188701/18870129/18870129_00000.pdf>, fecha de consulta [15/06/2011]

Alas Clarín, Leopoldo (1887b), «Los pazos de Ulloa (conclusión)», La Ilustración Ibérica 214, 6-7 [en línea], <http://hemerotecadigital.bne.es/datos1/numeros/ internet/Barcelona/Ilustración \% 20ibérica, \% 20La \% 20(Barcelona. \% 20 1883)/1887/188702/18870205/18870205_00000.pdf>, fecha de consulta [15/06/2011].

Alfonso, Luis (1887), «Cuentas atrasadas II», La Dinastía 2293, 2 [en línea], <http:/ / hemerotecadigital.bne.es/datos1/numeros/internet/Barcelona/Dinastía,\%20 La\%20(Barcelona)/1887/188708/18870805/18870805_00000.pdf>, fecha de consulta [15/06/2011].

ANÓNIMO (1882), «París», El Imparcial 5263, 4 [en línea], <http:/ / hemerotecadigital. bne.es/datos1/numeros/internet/Madrid/Imparcial,\%20El\%20(Madrid. \%20 1867)/1882/188201/18820130/18820130_00000.pdf>, fecha de consulta [15/06/2011]

ANÓNIMO (1885), «Bibliografía», El Imparcial 6620, 4 [en línea], <http:// hemerotecadigital.bne.es/datos1/numeros/internet/Madrid/Imparcial, \%20 El\%20(Madrid.\%201867)/1885/188511/18851102/18851102_00000.pdf>, fecha de consulta [15/06/2011]

Malagarriga, Carlos (1888), «La última obra de Zola», El Imparcial 7651, 5 [en línea], <http://hemerotecadigital.bne.es/datos1/ numeros / internet / Madrid / Imparcial, \% 20 E1 \% 20 (Madrid.\% 20 1867)/1888/188809/18880909/18880909_00000.pdf>, fecha de consulta [15/06/2011].

MoyA, Miguel (1886), «Los pazos de Ulloa», El Liberal 2701, 1-2 [en línea], <http:/ / hemerotecadigital.bne.es/datos1/numeros/internet/Madrid/Liberal, \% 20 El\%20(Madrid.\%201879)/1886/188610/18861028/18861028_00000.pdf>, fecha de consulta [15/06/2011].

Ortega Munilla, José (1890), «La última novela de Zola», El Imparcial 8211, 3 [en línea], <http://hemerotecadigital.bne.es/datos1/ numeros / internet / Madrid / Imparcial, \% 20E1 \% 20 (Madrid. \% 20 1867)/1890/189004/18900421/18900421_00000.pdf>, fecha de consulta [15/06/2011].

Pardo BazÁn, Emilia (1989), La cuestión palpitante (ed. GonzÁlez Herrán, José Manuel ), Barcelona: Anthropos.

Pattison, Walter. T. (1969), El naturalismo español: historia externa de un movimiento literario, Madrid: Gredos.

Picón, Jacinto Octavio (1883), «La última novela de Zola», El Imparcial 5671, 4 [en línea], <http://hemerotecadigital.bne.es/datos1/ numeros / internet / Madrid / Imparcial, \% 20 E1\% 20 (Madrid. \% 20 1867)/1883/188303/18830319/18830319_00000.pdf $>$, fecha de consulta [15/06/2011]. 
Segade Campoamor, Ramón (1887), «Los pazos de Ulloa», Galicia 1, 4555 [en línea], <http://hemerotecadigital.bne.es/datos1/numeros/ internet / A \% 20Coru \% C3\% B1a / Galicia \% 20(La \% 20Coru \% C3\% B1a. \% 20 1887)/1887/188701/18870100/18870100_00000.pdf>, fecha de consulta [15/06/2011].

\author{
František Dratva \\ Facultad de Filosofía y Letras \\ Universidad de Extremadura \\ Av. Universidad $\mathrm{s} / \mathrm{n}$ \\ Cáceres \\ 10071 \\ España \\ dratva1@seznam.cz
}

